\title{
Experimental Comparison of Bioinspired Segmentation Algorithms Applied to Segmentation of Digital Mammographies
}

\author{
David González-Patiño ${ }^{1}$, Yenny Villuendas-Rey², Amadeo J. Argüelles-Cruz ${ }^{1}$ \\ ${ }^{1}$ Centro de Investigación en Computación, Instituto Politécnico Nacional, \\ Ciudad de México, Mexico \\ ${ }^{2}$ Centro de Innovación y Desarrollo Tecnológico en Cómputo, Instituto Politécnico Nacional, \\ Ciudad de México, Mexico
}

davidglezp-92@hotmail.com, yenny.villuendas@gmail.com, aarguelles@ipn.mx

\begin{abstract}
Breast cancer has affected people worldwide despite of age or economic condition. In this paper, we explore the possibility of using bioinspired algorithms, mainly designed for optimization, for segmentation of mammographies, which showed good performances in this task. The results showed useful methods with promising results to segment digital mammographies using bioinspired algorithms.
\end{abstract}

Keywords: breast cancer, bioinspired algorithm, comparison, segmentation optimization.

\section{Introduction}

Breast cancer is one of the main causes of death in women all over the world [1]. This is the main reason why researchers have been trying to detect breast cancer in early phases using non-invasive techniques instead of invasive ones such as biopsies.

Mammographies studies are a useful tool for medics in order to diagnose breast cancer in early phases since it allows them to get a digital image of the breast using a low dose of x-rays. These studies are recommended to be done for women older than 26 years once a year [1].

A mammography is a digital image produced by a mammographic study and typically, these images are gray scales which allows to differentiate the lesion in the breast due to the texture and color [2]. To perform this task, the medic needs to segment the region of interest (lesion) in order to split lesion and background and diagnose accurately.

The bioinspired algorithm are inspired, as referred by their name, in natural and biological processes and they have been used to solve optimization problems [3]; however this has not been the unique approach where they have been used. 
In this paper, we perform an experimental comparison using bioinspired algorithms to segment mammography images and comparing the results with other algorithms used for segmentation.

The paper is organized as follows. Section 2 is a review of some of the previous works in automatic or semi-automatic segmentation processes in breast cancer mammographies. In section 3, there is a little explanation of the algorithms used in this work. Section 4 presents the results of the experiments got by the algorithms using them in segmentation of mammography images and finally in Section 5, the conclusions and future work is presented.

\section{Previous Works}

Mammographies are not the unique tool to help the expert medic to diagnose breast cancer; however it has been the most commonly used technique.

Segmentation is a commonly used process after the mammography is taken, since it allows to split the digital image into groups of pixels which are easier to analyze [4].

Although, there are many segmentation algorithms, which have showed good performances; there is not a perfect algorithm for segmentation since many approaches have been developed according to the necessities of each problem.

Some algorithms have been used for automatic diagnosis of breast cancer such as the model proposed by Pena-Reyes and Sipper in 1999 [5] or the algorithm proposed by Yan et al. in 2003 [6], which uses local entropy to segment images.

Timp and Karssemeijer [7], proposed in 2004 a segmentation method based on dynamic programming to segment mammographies; this method showed to be statistically significant between that method and other existing methods.

Cascio et al. [8] proposed an algorithm for mammogram segmentation using neural networks. This algorithm also uses feature selection based on linear discriminating analysis to choose the features which are able to distinguish lesions.

In 2012 [9], a method to split a mammographic image in lesion and background was proposed; this algorithm was based on a maximum likelihood active contour which showed good performances.

Later on, in 2016 [10], Dubrovina et al. presented a method using deep neural networks for segment mammographies and in the same year, Sargent and Park [11] implemented a method to segment mammographies using side-by-side comparisons.

Most of the works only focus on comparisons between classic algorithms, and, bioinspired algorithms have not been compared with classic algorithms to segment, this is why we addressed this problem in this paper.

\section{Segmentation Algorithms}

In this work, three algorithms were tested, a classic algorithm named Otsu method, which is very used in segmentation tasks. The remaining two algorithms are genetic algorithms and simulated annealing inspired in two different processes. 
Otsu method [12] is a thresholding method based on selecting the optimal limit to segment images into two regions. This method has been widely used in many segmentation problems showing good performances. Even so, this method have problems when one of the regions is significantly bigger, however this problem has been solved in newer implementations of this method.

Genetic algorithms (GA) are a subclass of evolutionary algorithms which are heuristics inspired on natural selection theory [13]. These algorithms generate random populations at the beginning and improve their individuals each generation through crossover and mutation in order to keep the best population according to a fitness function.

Simulated Annealing (SA) [14] is a technique used to find the global optimum which relies on the principle of annealing in metallurgy which involves heating and control the cooling phase in order to reduce the defects.

Since most of the bioinspired algorithms are focused for optimization problems, in this work there was a necessity to change the approach.

For this task, the Dunn index was used [15]; this index allows to evaluate clustering algorithms, so, the higher the Dunn index, the better the clustering.

To represent a possible solution for the bioinspired algorithms in this work, we used the representation of the individuals showed in figure 1.

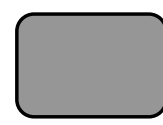

150



50

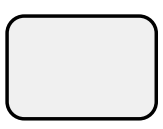

240

Fig. 1. Representation of an individual.

This representation shows an individual with 3 components, which components are represented as gray levels in the gray scale.

Table 1. Description of variables for equations 1 and 2.

\begin{tabular}{cl}
\hline Variable & \multicolumn{1}{c}{ Description } \\
\hline $\boldsymbol{N}$ & Number of regions \\
$\boldsymbol{S j}$ & Quantity of pixels in region $\mathrm{j}$ \\
$\boldsymbol{S I}$ & Area of the image \\
$\boldsymbol{N}(\boldsymbol{b})$ & Number of regions with exactly an area equal to b units \\
$\boldsymbol{e} \boldsymbol{j}^{2}$ & Squared color error of region $\mathrm{j}$ \\
$\boldsymbol{C x}(\boldsymbol{p})$ & Value of component x for pixel $\mathrm{p}$ \\
$\boldsymbol{C x}(\boldsymbol{R} \boldsymbol{j})$ & Average value of component $\mathrm{x}$ in the region $\mathrm{j}$ \\
\hline
\end{tabular}

To evaluate the segmentation done by each algorithm, we used the error measures presented by Hui Zhang et al. in 2007 [16]. These measures were presented for image segmentations using unsupervised methods. The two measures used were Average squared color error, defined as F (equation 1) and F' (equation 2), which is an improved version of $\mathrm{F}$ which penalizes segmentations which contains lots of small regions: 


$$
\begin{gathered}
\mathrm{F}=\sqrt{N} \sum_{j=1}^{N} \frac{e j^{2}}{\sqrt{s j}}, \\
\mathrm{~F}^{\prime}=\frac{1}{1000 * S I} \sqrt{\sum_{b=1}^{M a x} \operatorname{Area} N(b)^{1+\frac{1}{b}}} \sum_{j=1}^{N} \frac{e j^{2}}{\sqrt{S j} .}
\end{gathered}
$$

The variables for each equation are defined in table 1 .

\section{$4 \quad$ Experiments}

The images used in this work were provided by the Faculty of Medicine of the University of Porto, Portugal in the dataset named Breast Cancer Digital Repository [17], which is a repository that contains 200 lesions of 190 Portuguese women, corresponding to 362 segmentations of mammographies identified by expert radiologists.

The images are described by 38 attributes, representing several characteristics of the lesions, such as clinical data, descriptors of the intensity of the lesion contour, descriptors of the lesion texture and descriptors of location and shape of the lesion. In addition, each image has associated the corresponding class (malign, benign). These classes were corroborated by biopsy analysis, that is, we are certain about the classification of each lesion.

The dataset contains some missing values (represented by NaN, Not a Number), which were substituted by the attribute mean.

The three algorithms presented in this work processed all the images, and the error measures were calculated for each image. The results are presented in this section.

Tables 2-4 show the segmentation of three lesions, as an example. In these tables, we show the original image carried out by an expert radiologist, the lesion segmented by the radiologist (Region of Interest, ROI), the ROI overlapped with the mammography image, and then, the segmentation obtained by Otsu method, Genetic Algorithm (GA) and Simulated Annealing (SA), respectively.

As shown, the algorithm that got the best segmentation was genetic algorithms, eventhough the region of interest is not covered as much as in the other images, the region which does not belong to the region of interest is not as much as in the other two algorithms.

In the images is observed that Otsu method segmented the region of interest with a bigger area compared to the other segmented images. This is relevant since the other algorithms got a lower area and they had a bigger similarity to the original segmented image carried out by an expert radiologist.

Genetic algorithms got a better segmentation comparing the produced image with the original image carried out by an expert radiologist since the area in the image is similar to the original segmented image carried out by an expert radiologist.

In this experiment can be observed that the three segmented images are visually similar to the original segmented image carried out by an expert radiologist. Eventhough the error measures in this image could be similar, the values for all the images need to be calculated in order to obtain correct conclusions. 
Experimental Comparison of Bioinspired Segmentation Algorithms Applied to Segmentation ...

Table 2. (a) Original image, (b) ROI segmented by expert radiologist, (c) Overlap of the ROI over original image, (d) Image segmented by Otsu method, (e) Image segmented by GA, (f) Image segmented by SA.



Table 3. (a) Original image, (b) ROI segmented by expert radiologist, (c) Overlap of the ROI over original image, (d) Image segmented by Otsu method, (e) Image segmented by GA, (f) Image segmented by SA.




Table 4. (a) Original image, (b) ROI segmented by expert radiologist, (c) Overlap of the ROI over original image, (d) Image segmented by Otsu method, (e) Image segmented by GA, (f) Image segmented by SA.

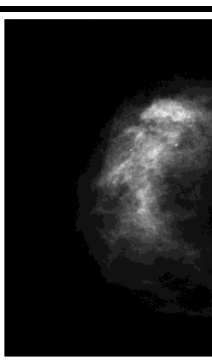

(a)

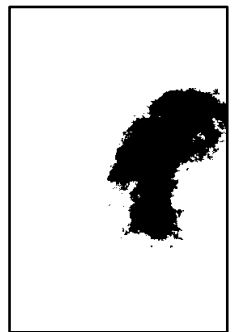

(d)

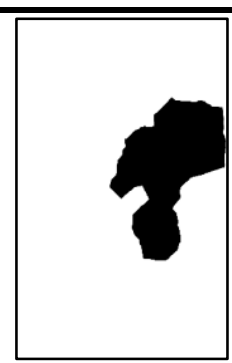

(b)

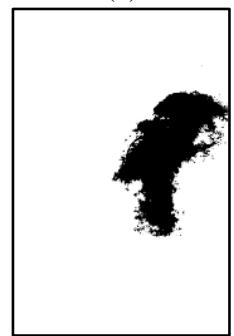

(e)

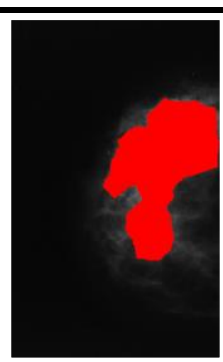

(c)

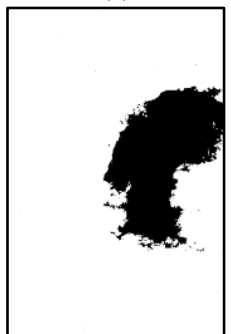

(f)

All the 362 images were processed and the error measures were calculated for each image by equations 1 and 2 . The table 5 shows the measures calculated.

Table 5. Mean of the errors calculated for the segmentation of the 362 mammographies.

\begin{tabular}{ccc}
\hline Algorithm & F mean & F' mean \\
\hline Otsu method & $15.324 \times 10^{6}$ & $12.880 \times 10^{-3}$ \\
Genetic Algorithm & $9.888 \times 10^{6}$ & $8.313 \times 10^{-3}$ \\
Simulated annealing & $14.88 \times 10^{6}$ & $12.519 \times 10^{-3}$ \\
\hline
\end{tabular}

In addition, a comparison in running time was done for the 3 algorithms and the results of the mean times are presented in table 6 .

Table 6. Times calculated for the segmentation of the 362 mammographies.

\begin{tabular}{cccc}
\hline Algorithm & Mean time (secs.) & $\begin{array}{c}\text { Minimum time } \\
\text { (secs.) }\end{array}$ & $\begin{array}{c}\text { Max. time } \\
\text { (secs.) }\end{array}$ \\
\hline Otsu method & 0.0515 & 0.022 & 0.590 \\
Genetic Algorithm & 170.830 & 126 & 278 \\
Simulated annealing & 130.406 & 97 & 211 \\
\hline
\end{tabular}

As shown in tables 5 and 6, the algorithm having the best segmentation was Genetic algorithm since the mean error calculated was lower compared to the other algorithms. 
Experimental Comparison of Bioinspired Segmentation Algorithms Applied to Segmentation ...

This could be due to the exploration and the capabilities of the algorithm for finding better solutions which produce better segmentations.

Simulated annealing got a higher error compared with genetic algorithm, however the error presented in Simmulated annealing is lower than the one calculated for Otsu method.

With respect to computational cost Otsu method showed a very lower time compared to the other algorithms, which showed to be a good implementation and a fast method to produce binary images with the possibility to segment the region of interest.

\section{Conclusions}

The bioinspired algorithms tested in this work showed lower errors comparing them to Otsu method, which is a very used method for thresholding. Although the genetic algorithm got a lower error and Otsu got the highest error, in the running time test, the genetic algorithm got the highest running times while the Otsu method got very little running times.

This could be due to the implementation of the Otsu method in Matlab since this algorithm is implemented and optimized in order to execute faster operations.

According to the error measures calculated, Genetic algorithms got a lower error when segmenting the mammography images, this can be visually observed in the segmented images since the regions of the segmented images are smaller and cover a better portion of the original segmented image carried out by an expert radiologist.

In this comparison the best algorithm applied for segmentation was Genetic algorithms according to the error measures, although Otsu got the lowest running time.

Acknowledgements. The authors would like to thank the Instituto Politécnico Nacional (Secretaría Académica, Comisión de Operación y Fomento de Actividades Académicas, Secretaría de Investigación y Posgrado, Centro de Investigación en Computación, and Centro de Innovación y Desarrollo Tecnológico en Cómputo), the Consejo Nacional de Ciencia y Tecnología (Conacyt), and Sistema Nacional de Investigadores in México for their economical support to develop this work. Also thanks to the support provided by the SIP project 20170362, for the economic support and related work.

\section{References}

1. Ferlay, J., Héry, C., Autier, P., Sankaranarayanan, R.: Global burden of breast cancer. In: Breast cancer epidemiology. pp. 1-19. Springer (2010)

2. Yaffe, M.J.: Digital mammography. In: PACS. pp. 363-371 (2006)

3. Yang, X.-S., Hossein Gandomi, A.: Bat algorithm: a novel approach for global engineering optimization. Eng. Comput. 29, 464-483 (2012)

4. Pham, D.L., Xu, C., Prince, J.L.: Current methods in medical image segmentation 1. Annu. Rev. Biomed. Eng. 2, 315-337 (2000) 
5. Pena-Reyes, C.A., Sipper, M.: A fuzzy-genetic approach to breast cancer diagnosis. Artif. Intell. Med. 17, 131-155 (1999)

6. Yan, C., Sang, N., Zhang, T.: Local entropy-based transition region extraction and thresholding. Pattern Recognit. Lett. 24, 2935-2941 (2003)

7. Timp, S., Karssemeijer, N.: A new $2 \mathrm{D}$ segmentation method based on dynamic programming applied to computer aided detection in mammography. Med. Phys. 31, 958$971(2004)$

8. Cascio, D., Fauci, F., Magro, R., Raso, G., Bellotti, R., De Carlo, F., Tangaro, S., De Nunzio, G., Quarta, M., Forni, G., others: Mammogram segmentation by contour searching and mass lesions classification with neural network. IEEE Trans. Nucl. Sci. 53, 2827-2833 (2006)

9. Rahmati, P., Adler, A., Hamarneh, G.: Mammography segmentation with maximum likelihood active contours. Med. Image Anal. 16, 1167-1186 (2012)

10. Dubrovina, A., Kisilev, P., Ginsburg, B., Hashoul, S., Kimmel, R.: Computational mammography using deep neural networks. Comput. Methods Biomech. Biomed. Eng. Imaging Vis. 1-5 (2016)

11. Sargent, D., Park, S.Y.: Automatic segmentation of mammogram and tomosynthesis images. In: SPIE Medical Imaging. (2016)

12. Otsu, N.: A threshold selection method from gray-level histograms. Automatica 11, 23-27 (1975)

13. Banzhaf, W., Nordin, P., Keller, R.E., Francone, F.D.: Genetic programming: an introduction. Morgan Kaufmann San Francisco (1998)

14. Khachaturyan, A.G., Semenovskaya, S. V, Vainstein, B.: A statistical-thermodynamic approach to determination of structure amplitude phases. Sov. Phys. Crystallogr. 24, 519 524 (1979)

15. Dunn, J.C.: A fuzzy relative of the ISODATA process and its use in detecting compact well-separated clusters. (1973)

16. Zhang, H., Fritts, J.E., Goldman, S.A.: Image segmentation evaluation: A survey of unsupervised methods. Comput. Vis. image Underst. 110, 260-280 (2008)

17. Moura, D.C., López, M.A.G.: An evaluation of image descriptors combined with clinical data for breast cancer diagnosis. Int. J. Comput. Assist. Radiol. Surg. 8, 561-574 (2013) 